\title{
Response to Comment by C. H. Lee, P. Dutilleul and A. Roy on "Models with a Kronecker Product Covariance Structure: Estimation and Testing"
}

\author{
M. S. Srivastava, T. von Rosen, and D. von Rosen \\ Received November 25, 2009
}

DOI: $10.3103 / \mathrm{S} 1066530710010072$

We are grateful to Lee, Dutilleul \& Roy for their written comments to the Editor of the journal, Mathematical Methods of Statistics, on our 2008 paper, in which they raised some questions regarding some of our statements in the above mentioned paper. We believe it arose from a misunderstanding of the fact that it is not necessarily true that all estimators obtained from the likelihood equations, even when all the parameters are uniquely defined, are unique. This is not true unless the model belongs to the regular exponential family. There are no incorrect statements in the paper except that the reference to the paper by Roy \& Khattree (2005) should have come later than in the beginning. It may be noted that the estimate of $\rho$ obtained from the likelihood equations may not guarantee that it will be positive. One may truncate it but then it may not be unique.

The flip-flop algorithm is not new in statistics except maybe its naming. For example, the maximum likelihood estimators of the well-known Growth curve model introduced by Potthoff \& Roy (1964) were obtained in this fashion until Khattri (1966) obtained it explicitly, i.e. one went back and forth between the equations obtained from differentiating the mean and differentiating the dispersion. The suggestion that the likelihood of the observations for this model is invariant under scalar transformation and thus one can obtain estimates of $\Psi \otimes \Sigma$ first and then put the condition that one element of any of these two matrices is known will give unique estimators requires a proof, that is what has been done in our paper under discussion. Besides, convergence problems may occur since the elements of one matrix can be made as large as we please by making the elements of the other matrix small; this may cause convergence problems in iterative procedures. 